Portland State University

PDXScholar

$10-1-2001$

\title{
Dynamical theory for modeling dipole-dipole interactions in a microcavity: The Green dyadic approach
}

\author{
P.T. Leung \\ Portland State University \\ R. L. Hartman
}

Follow this and additional works at: https://pdxscholar.library.pdx.edu/phy_fac

Part of the Physics Commons

Let us know how access to this document benefits you.

\section{Citation Details}

Hartman, R. L., \& Leung, P. T. (2001). Dynamical theory for modeling dipole-dipole interactions in a microcavity: The Green dyadic approach. Physical Review B (Condensed Matter And Materials Physics), 64(19), 193308/1-4.

This Article is brought to you for free and open access. It has been accepted for inclusion in Physics Faculty Publications and Presentations by an authorized administrator of PDXScholar. Please contact us if we can make this document more accessible: pdxscholar@pdx.edu. 


\title{
Dynamical theory for modeling dipole-dipole interactions in a microcavity: The Green dyadic approach
}

\author{
R. L. Hartman and P. T. Leung \\ Department of Physics, Portland State University, P.O. Box 751, Portland, Oregon 97207-0751
}

(Received 29 May 2001; published 19 October 2001)

\begin{abstract}
A dynamical theory for modeling the dipole-dipole interaction in a microcavity is formulated using the Green dyadic approach. To our knowledge, this theory is one of the most general in many aspects of modeling the phenomenon. It accommodates an arbitrary number of layers adjacent to the cavity, constant but arbitrary dielectric properties within each layer, inclusion of retardation effects, arbitrary dipole orientations, and an unlimited number of interacting dipoles. Numerical results for the emission properties of interacting molecular dipoles in a microcavity are presented to illustrate the capability of the method.
\end{abstract}

DOI: 10.1103/PhysRevB.64.193308

PACS number(s): 41.20.- q, 42.50.Fx

\section{INTRODUCTION}

It has been well known that molecular emission properties can be significantly modified in the vicinity of a surface or inside a microcavity. ${ }^{1}$ For example, recent studies have shown that significant control of spontaneous emission ${ }^{2}$ and Raman-scattering enhancement ${ }^{3}$ can be achieved from emitting dipoles confined in planar metallic and semiconducting, as well as dielectric microcavities. Theoretical studies of this phenomenon have been extensive, including both classical and quantum mechanical modeling, for both well-defined (planar, spherical, etc.) and arbitrary geometrical boundaries. ${ }^{4-8}$ Moreover, these modeling studies have considered most of the time a single (molecular) dipole interacting with the multistack "environment."

Besides modified dipolar emission characteristics, recent experimental studies have also shown significant surface or cavity-induced effects on the dipole-dipole interaction between the molecules or particles confined to such a proximity. These studies include the observation of the surfacemode-modified dipole-dipole interaction among adsorbed silver nanoparticles, ${ }^{9}$ that of the enhanced energy-transfer process between donors and acceptors, ${ }^{10}$ and that of the unambiguous confirmation of enhanced nonradiative Forster transfer between molecules confined in planar microcavities. ${ }^{11}$ As Barnes and Andrew explained in their commentary, ${ }^{12}$ such control of energy transfer is of high significance and may lead to many and varied applications in areas as diversified as photochemistry and optoelectronics.

There has also been a large number of theoretical works on the modeling of dipole-dipole interaction for molecules confined in the vicinity of planar surface or microcavities. However, due to the complexity of the dynamics and geometrical boundaries, most of these previous works were limited in some aspects. These limitations include, for example, (i) the assumption of perfect conducting ${ }^{13}$ or realistic (but symmetrical) boundaries ${ }^{14}$ for the microcavity, (ii) the limitation to a single medium (of infinite extent) on each side of the microcavity, ${ }^{13,14}$ and (iii) the modeling of a single pair of interacting dipoles. ${ }^{13,14}$ In addition, as pointed out in Ref. 10, the total dynamical (retarded) dipole-dipole interaction has not been fully accounted for in these previous works.
It is the purpose of this work to present a relatively powerful method for the modeling of the interaction among the molecular dipoles confined in a planar microcavity or photonic band-gap material structure. This method will allow the modeling in principle to incorporate any number of interacting dipoles in arbitrary orientations, any number of "adjacent layers" of realistic dielectric properties on each side of the microcavity, and the full incorporation of retardation effects. We will present the method and demonstrate its applicability via numerical computations with respect to the geometry used in the experiment in Ref. 10. Though our following theory is based on a classical phenomenological approach, yet it is well known that this approach is as accurate as a quantum-mechanical approach as long as one is interested only in emission properties normalized to the free decay rates of the molecules. ${ }^{4}$

\section{THEORY}

Let us refer to the geometry of Ref. 10 as depicted in Fig. 1 , where a number of molecular dipoles are embedded in a microcavity which is formed by a multistack reflector at the bottom and a metallic superstrate at the top. Our approach is to use the Green dyadic for solving Maxwell's equations for such a multilayer system which has been derived previously. ${ }^{15}$ The calculation of the Green dyadic for a multilayer system is a problem of high significance and has been investigated extensively in the literature. ${ }^{16}$ The work we base on has reformulated this problem in a slightly simpler fashion, leading to more efficient numerical implementation. Briefly, the dyadic for a multilayer system can be expressed as follows: ${ }^{15}$

$$
\mathbf{G}\left(\mathbf{R}, \mathbf{R}^{\prime}\right)=\delta(j, s) \mathbf{G}_{s o}\left(\mathbf{R}, \mathbf{R}^{\prime}\right)+\mathbf{G}_{j}\left(\mathbf{R}, \mathbf{R}^{\prime}\right)
$$

where $\mathbf{R}$ is contained in slab $j$, the current source at $\mathbf{R}^{\prime}$ is contained in slab $s$, and $\delta(j, s)$ is the Kronecker delta. The source term $\mathbf{G}_{s o}\left(\mathbf{R}, \mathbf{R}^{\prime}\right)$ for a source in slab $s$ takes the wellknown form ${ }^{17}$ 


$$
\begin{aligned}
\mathbf{G}_{s o}\left(\mathbf{R}, \mathbf{R}^{\prime}\right)= & \frac{-1}{k_{s}^{2}} \hat{z} \hat{z}^{t} \delta\left(\mathbf{R}-\mathbf{R}^{\prime}\right) \\
& +\frac{i}{4 \pi} \int_{0}^{+\infty} d \lambda \sum_{n=0}^{+\infty} \frac{2-\delta(n, 0)}{\lambda h_{s}(\lambda)} \sum_{l=0}^{1}\left(\begin{array}{l}
\mathbf{M}_{l, n, \lambda}\left(+h_{s}\right) \mathbf{M}_{l, n, \lambda}^{\prime t}\left(-h_{s}\right)+\mathbf{N}_{l, n, \lambda}\left(+h_{s}\right) \mathbf{N}_{l, n, \lambda}^{\prime t}\left(-h_{s}\right) \\
\mathbf{M}_{l, n, \lambda}\left(-h_{s}\right) \mathbf{M}_{l, n, \lambda}^{\prime t}\left(+h_{s}\right)+\mathbf{N}_{l, n, \lambda}\left(-h_{s}\right) \mathbf{N}_{l, n, \lambda}^{\prime t}\left(+h_{s}\right)
\end{array}\right) \begin{array}{l}
z \leqslant z^{\prime} \\
z \leqslant z^{\prime}
\end{array}
\end{aligned}
$$

where the prime indicates $\mathbf{R}^{\prime}$ dependence, the absence of a prime indicates $\mathbf{R}$ dependence, and $t$ indicates matrix transpose. The functions $h_{j}=\sqrt{k_{j}^{2}-\lambda^{2}}$, and that $\mathbf{M}$ and $\mathbf{N}$ are defined as before, ${ }^{4,17}$ where $k_{j}=\omega \sqrt{\mu_{j} \varepsilon_{j}}$ denotes the wave number. In the previous approach to calculate the scattering part of $\mathbf{G}$ in Eq. (1), we have carried out the expansion by reassociating it with the source current as follows: ${ }^{15}$

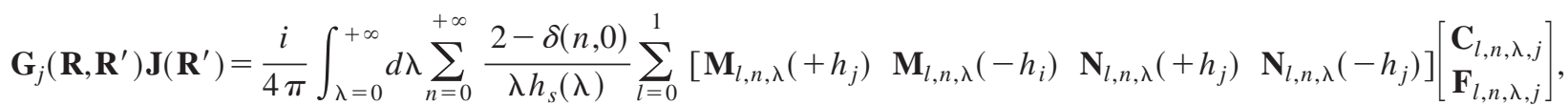

where

$$
\begin{gathered}
\mathbf{C}_{l, n, \lambda, j} \equiv\left[\begin{array}{l}
c_{l, n, \lambda, j} \\
c_{l, n, \lambda, j}^{\prime}
\end{array}\right], \\
\mathbf{F}_{l, n, \lambda, j} \equiv\left[\begin{array}{l}
f_{l, n, \lambda, j} \\
f_{l, n, \lambda, j}^{\prime}
\end{array}\right],
\end{gathered}
$$

and the radiation boundary condition requires that, at the slabs $j=0$ and $j=N$,

$$
c_{l, n, \lambda, 0}=c_{l, n, \lambda, N}^{\prime}=f_{l, n, \lambda, 0}=f_{l, n, \lambda, N}^{\prime}=0 .
$$

Note that square-brackets will be used to exhibit matrices in terms of their entries and that Eq. (3) contains products of $3 \times 4$ and $4 \times 1$ matrices. Also note that the vectors $\mathbf{M}$ and $\mathbf{N}$ in Eq. (3) are functions of $\mathbf{R}$, whereas $\mathbf{C}_{l, n, \lambda, j}$ and $\mathbf{F}_{l, n, \lambda, j}$ depend on both $\mathbf{R}$ and $\mathbf{R}^{\prime}$. By matching the boundary con-

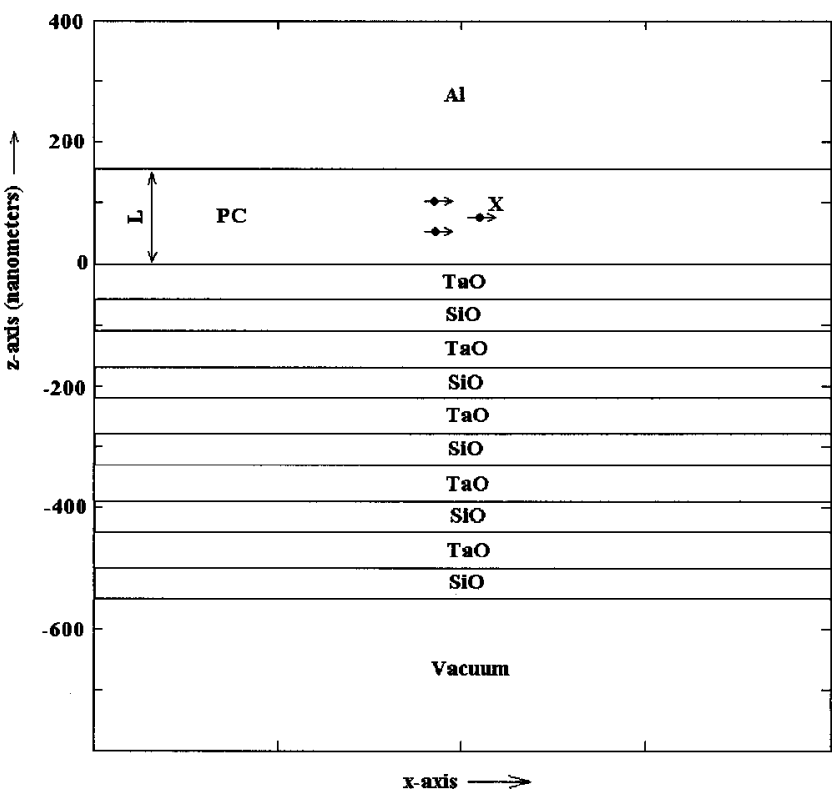

FIG. 1. Geometry of the multistack planar microcavity according to Ref. 10 . The cavity dimension $L$ and the designated dipole $X$ are as labeled. ditions for the transverse fields, i.e., the continuity of $\hat{\mathbf{z}}$ $\times \mathbf{E}(\mathbf{R})$ and $\hat{\mathbf{z}} \times \boldsymbol{\nabla} \times \mathbf{E}(\mathbf{R})$ at each interface, the coefficients $\left[\begin{array}{l}\mathbf{C}_{l, n, \lambda, j} \\ \mathbf{F}_{l, n, \lambda, j}\end{array}\right]$ can be obtained through some recursion relations. ${ }^{15}$ Note that the arbitrary current source $\mathbf{J}$ is also contained in $\left[\begin{array}{l}\mathbf{C}_{l, n, \lambda, j} \\ \mathbf{F}_{l, n, \lambda, j}\end{array}\right]$ so that $\mathbf{G}_{j}$ can be extracted from Eq. (3).

To apply the above result for the dyadic to our modeling of the dipole-dipole interaction in the geometry of Fig. 1, let us focus on one of the emitting molecular dipoles (dipole $X$ ) in the cavity. According to the classical phenomenological approach, ${ }^{4}$ the frequency shift and decay rate of this dipole, normalized to the free decay value, can be obtained in terms of the real and imaginary parts of the total (excluding its own) field $\mathbf{E}$ acting at the dipole site as (in SI units):

$$
\begin{aligned}
& \frac{\Delta \omega}{\gamma_{0}}=-\frac{3 \pi \varepsilon_{0} q n_{s}^{2}}{p_{0} k_{s}^{3}} \operatorname{Re}(\mathbf{E}), \\
& \frac{\gamma}{\gamma_{0}}=1+\frac{6 \pi \varepsilon_{0} q n_{s}^{2}}{p_{0} k_{s}^{3}} \operatorname{Im}(\mathbf{E}),
\end{aligned}
$$

where $q$ is the intrinsic quantum yield and $k_{s}=n_{s} \omega / c$, with $n_{s}$ the real refractive index of the medium containing the dipole. Note that $p_{0}$ and $\omega$ are the dipole moment and emission frequency of the molecule, and the only quantity needed to be calculated in this model is the field acting on $X$, which can be obtained from the Green dyadic equations (1)-(3) of the problem as follows:

$$
\mathbf{E}\left(\mathbf{R}_{x}\right)=i \omega \mu \int \mathbf{G}\left(\mathbf{R}_{x}, \mathbf{R}^{\prime}\right) \mathbf{J}\left(\mathbf{R}^{\prime}\right) d V\left(\mathbf{R}^{\prime}\right)
$$

where $\mu$ is the magnetic permeability for the vacuum, where we have restricted ourselves to nonmagnetic media. To model our problem as described in Fig. 1, we write the current density in Eq. (7) in the form

$$
\mathbf{J}=-\sum_{i} i \omega \mathbf{p}_{i} \delta\left(\mathbf{R}^{\prime}-\mathbf{R}_{i}\right)
$$

where $\mathbf{p}_{i}$ is the molecular dipole moment located at $\mathbf{R}_{i}$. Note that a time dependence of the form $e^{-i \omega t} e^{-\gamma t / 2}$ has been 
Two Parallel Dipoles

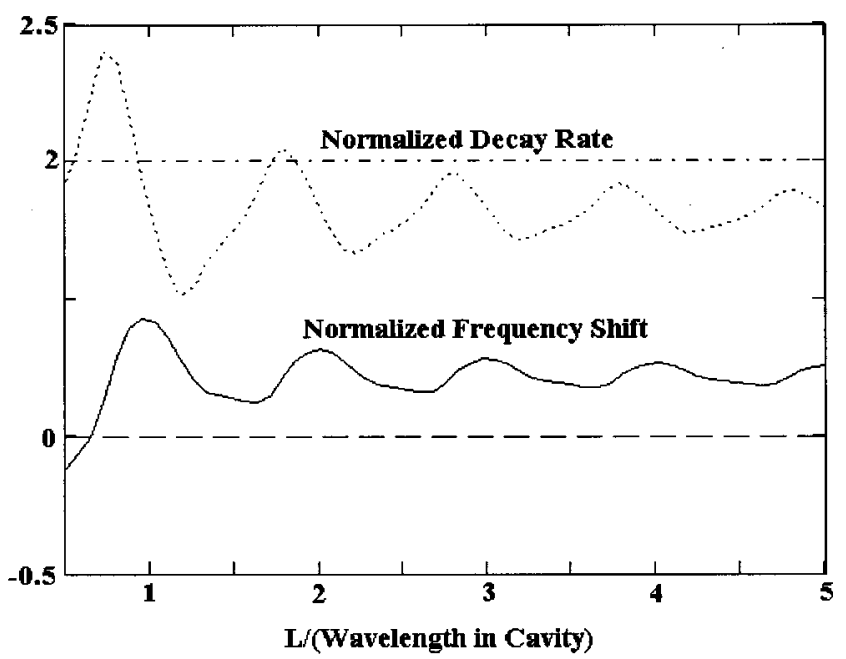

FIG. 2. Results for two parallel dipoles located at $(x, z)$ $=[0,(L+d) / 2]$ and $[0,(L-d) / 2]$, respectively, where $d$ $=($ emission wavelength in the PC medium $) / 2 \pi$. The one at $[0,(L$ $+d) / 2]$ is our designated dipole $X$.

assumed in Eqs. (7) and (8). Thus, using Eqs. (1)-(8) and excluding the "self-field" [i.e. contribution from $\mathbf{G}_{0}\left(\mathbf{R}_{x}, \mathbf{R}_{x}\right)$ in Eq. (2)], we can simulate the emission properties of the specific dipole $X$ in the microcavity. Note that this cooperative decay rate in Eq. (6) is directly linked to the energytransfer rate of $X$ to the other molecules as well as to the cavity environment, and the frequency-shift in Eq. (5) reveals the interaction energy between $X$ and the other molecules as well as the environment, when they are all confined by the microcavity geometry. ${ }^{18}$ Furthermore, Eq. (8) implies that this approach is very general and can include in principle an arbitrary number of interacting dipoles randomly oriented inside the microcavity.

\section{NUMERICAL RESULTS}

To demonstrate the capability of the above method, we have computed Eqs. (5) and (6) for the cases of two and three dipoles interacting in the microcavity (Fig. 1), respectively. For each case, the emission properties of the designated dipole $X$ are calculated as a function of cavity dimension $(L)$ with all other parameters fixed. The emission wavelength $(612 \mathrm{~nm})$ and the values for the dielectric constants for various materials of the cavity are used in accord with Ref. 10. Figures 2 and 3 show the results for the cases when the two dipoles are parallel and perpendicular to the cavity boundaries, respectively. From the results, the "cavity-resonance effect" can be clearly seen, and it is most obvious in the parallel dipole case. ${ }^{13}$ In addition, the frequency shifts of the designated dipole $X$, which reveals the dipole-dipole interaction within the cavity, are seen to depend drastically on the dipole orientations. In this case we obtain mostly blueshifts for parallel dipoles and redshifts for perpendicular dipoles. This happens since $X$ remains close to one of the boundaries and the result is dominated by its own
Two Perpendicular Dipoles

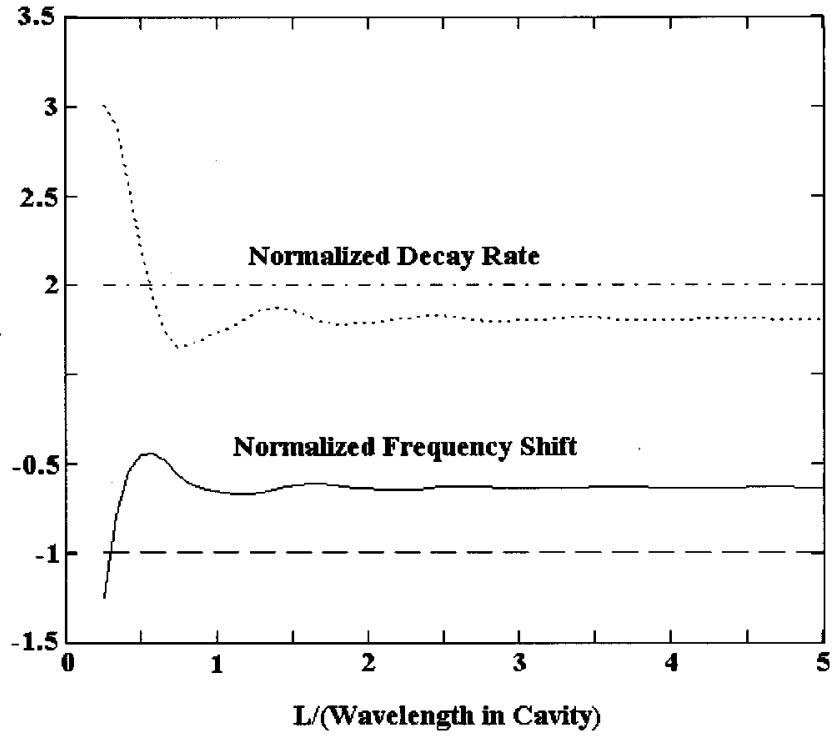

FIG. 3. Results for two perpendicular dipoles located at $(0, L / 2)$ and $(d, L / 2)$, respectively, with $X$ located at $(d, L / 2)$.

image fields from this boundary (recall that a parallel dipole has its image opposite while a perpendicular dipole has its image along its own orientation ${ }^{18}$ ). The result, however, is sensitive to the location of this dipole $X$ relative to the cavity boundary as well as to the other dipoles in the cavity. For example, Fig. 4 shows the results for three parallel dipoles with the $X$ dipole located at the middle of the cavity and off the axis joining the other two dipoles. In this case, we see that even for dipoles oriented parallel to the cavity boundaries, redshifts in their emission frequencies can result upon interaction with the other dipoles in the same cavity. We want to remark that while the incorporation of a large number of dipoles is rather straightforward (though computationtime consuming) in our present formalism, it is not clear if

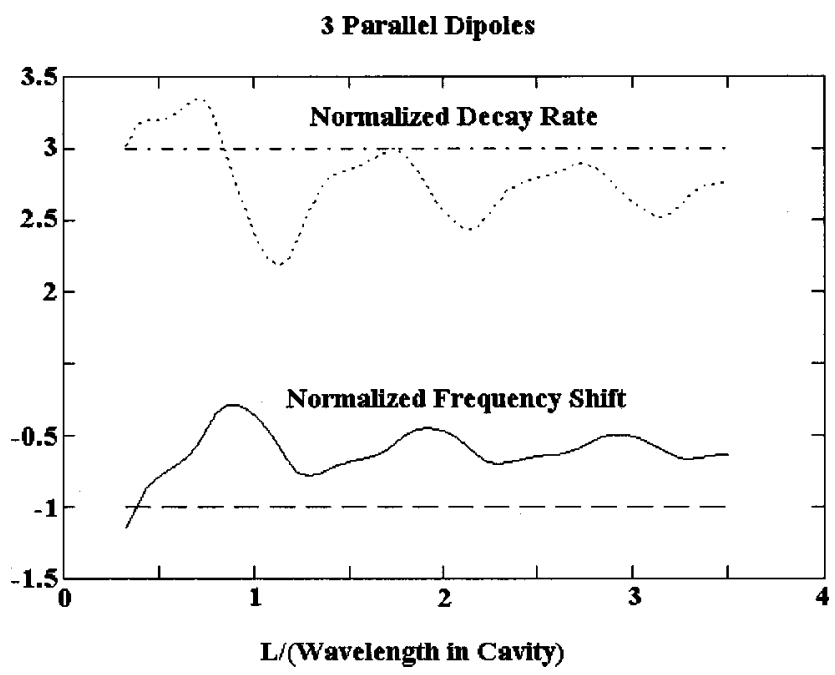

FIG. 4. Results for three parallel dipoles located at $[0,(L$ $+d) / 2],[0,(L-d) / 2]$, and $(d, L / 2)$, respectively, with $X$ located at $(d, L / 2)$. 
the same is true in the previous approaches appeared in the literature.

\section{CONCLUDING REMARKS}

We have thus in the above demonstrated how the Green dyadic solution for a multilayer system can be applied to model the interaction of a system of dipoles confined in a planar microcavity. In particular, we have illustrated how a complicated multistack structure and collection of dipoles can be systematically simulated in this approach. As is clear from the above, the results for the cooperative decay and frequency shift are very sensitive to the presence of the cavity and the other dipoles, consistent with the observations reported previously in the literature. ${ }^{10-14}$ Apparently, the response function approach of Agarwal and Gupta ${ }^{14}$ can also be generalized to such an extent for simulating an arbitrary multilayer of planar geometry and a collection of arbitrary dipoles, but it has yet to be carried out. At the completion of our work, it came to our attention that in a very recent paper, ${ }^{19}$ Bennett et al. have also applied the Green dyadic approach to simulate interacting dipoles (up to two dipoles) in a planar microcavity. However, their superlattice geometry is restricted to a periodical system of infinitely many layers, whereas our present formulation can accommodate a finite number of stratified layers of irregular thickness. Hence we believe our present approach has some usefulness in the modeling of actual experimental situations such as those in the experiment of Hopmeier et al. ${ }^{10}$

\section{ACKNOWLEDGMENT}

Partial support of this work has been provided by the Faculty Development Funding of Portland State University.
${ }^{1}$ See, e.g., Y. Yamamoto and R. E. Slusher, Phys. Today 46 (6), 66 (1993); W. L. Barnes, J. Mod. Opt. 45, 661 (1998).

${ }^{2}$ H. Rigneault et al., Phys. Rev. A 55, 1497 (1997); I. Abram et al., IEEE J. Quantum Electron. 34, 71 (1998); P. E. Burrows et al., Appl. Phys. Lett. 73, 435 (1998); S. Robert et al., J. Opt. Soc. Am. B 15, 1773 (1998).

${ }^{3}$ A. Fainstein and B. Jusserand, Phys. Rev. B 57, 2402 (1998).

${ }^{4}$ R. R. Chance, A. Prock, and R. Silbey, Adv. Chem. Phys. 37, 1 (1978).

${ }^{5}$ Barnes (Ref. 1).

${ }^{6}$ H. Rigneault and S. Monneret, Phys. Rev. A 54, 2356 (1996).

${ }^{7}$ J. K. Hwang, H. Y. Ryu, and Y. H. Lee, Phys. Rev. B 60, 4688 (1999).

${ }^{8}$ A. Rahmani, P. C. Chaumet, and F. de Fornet, Phys. Rev. A 63, 023819 (2001).

${ }^{9}$ H. R. Stuart and D. G. Hall, Phys. Rev. Lett. 80, 5663 (1998).

${ }^{10}$ M. Hopmeier, W. Guss, M. Deussen, E. O. Gobel, and R. F. Mahrt, Phys. Rev. Lett. 82, 4118 (1999).
${ }^{11}$ P. Andrew and W. L. Barnes, Science 290, 785 (2000).

${ }^{12}$ W. L. Barnes and P. Andrew, Nature (London) 400, 505 (1999).

${ }^{13}$ T. Kobayashi, Q. Zheng, and T. Sekiguchi, Phys. Lett. A 199, 21 (1995); Phys. Rev. A 52, 2835 (1995).

${ }^{14}$ G. S. Agarwal and S. D. Gupta, Phys. Rev. A 57, 667 (1998).

${ }^{15}$ R. L. Hartman, J. Opt. Soc. Am. A 17, 1067 (2000). See also, R. L. Hartman, S. M. Cohen, and P. T. Leung, J. Chem. Phys. 110, 2189 (1999); R. L. Hartman, P. T. Leung, and S. M. Cohen, J. Opt. Soc. Am. A 17, 933 (2000).

${ }^{16}$ See, e.g., G. P. dos S. Cavalcante et al., Radio Sci. 17, 503 (1982); L. W. Li et al., Int. J. Electron. 70, 803 (1991); M. Paulus et al., Phys. Rev. E 62, 5797 (2000).

${ }^{17}$ C.-T. Tai, Dyadic Green Function in Electromagnetic Theory (IEEE Press, New York, 1994).

${ }^{18}$ A. Sommerfeld, Ann. Phys. (Leipzig) 28, 665 (1909); Partial Differential Equations in Physics (Academic, New York, 1949).

${ }^{19}$ C. R. Bennett, J. B. Kirk, and M. Babiker, Phys. Rev. A 63, 063812 (2001). 